\title{
ECONOMÍA Y SOCIEDAD RURAL
}

\section{Opciones tecnológicas para el fortalecimiento de las capacidades de los productores en tierras de ladera. El caso de la mora en el departamento de \\ Caldas}

Tobasura Acuña Isaías ${ }^{1}$, Obando Franco Humberto², Moreno Chávez Fred Alberto $^{3}$, Morales Londoño Carmen Soledad ${ }^{4}$, Henao Castaño Angélica María ${ }^{5}$ ${ }^{1} \mathrm{IA}$. Doctor en Sociología del Medio Ambiente. ${ }^{2}$ Edafólogo, PhD en Suelos. ${ }^{3}$ IA. Magister en Ciencias Económicas. ${ }^{4}$ IA. Magister en Suelos y Aguas. Docentes de la Universidad de Caldas, Departamento de Desarrollo Rural y Recursos Naturales. ${ }^{5} \mathrm{MVZ}$, Estudiante Maestría en Sociedades Rurales. isaias.tobasura@ucaldas.edu.co

\section{RESUMEN}

Introducción. Desde los años 1950, el modelo de desarrollo agrario implementado en Colombia ha promovido el uso de tecnologías agrícolas, orientado a incrementar los ingresos y mejorar el bienestar de los productores. Bajo este supuesto, se aplicaron "paquetes tecnológicos", soportados en variedades de alto rendimiento. Dicha tecnología ha sido desarrollada por centros de investigación y transnacionales, y transferida a los productores, mediante sistemas de extensión conductistas. En esta perspectiva, han emergido formas de agricultura, como la agricultura de conservación, prácticas de conservación de agrodiversidad, coberturas verdes, rotación de cultivos, localización precisa de los insumos y disturbios mínimo del suelo, se vislumbran no solo como innovaciones para mejorar la sostenibilidad y competitividad de los sistemas de producción locales, sino como alternativas para desencadenar procesos de aprendizaje, empoderamiento y fortalecimiento de las capacidades de los productores. Objetivo. Fortalecer las capacidades productivas y organizativas de los actores de la cadena de la mora en tierras de "ladera", con énfasis en los productores agrícolas y los prestadores de los servicios de extensión y asistencia. Metodología. Se realizaron distintas actividades, métodos y técnicas de investigación participativa en fincas de agricultores, utilizando los conceptos de 
Fincas de Aprendizaje (FA) y Escuelas de Campo de Agricultores (ECAs). En cada municipio del proyecto se conformó una ECA, alrededor de la cual gravitó el proceso de construcción, uso y apropiación social del conocimiento. Resultados. Se ha logrado compartir de manera participativa innovaciones y prácticas tecnológicas que se han desarrollado en el programa "Manejo de suelos, agua y nutrientes para la producción sostenible de mora en el Departamento de Caldas", y en el proyecto "De la Transferencia de Tecnología a la Mediación Participativa. Agricultura de Conservación en Suelos de Ladera del Departamento de Caldas (Colombia)". Conclusiones. Los resultados del proceso de investigación/ extensión han contribuido a enriquecer los fundamentos teóricos y las prácticas de cultivo entre investigadores, técnicos y productores, los cuales han permitido incrementar el rendimiento de los cultivos, racionalizar los costos de producción, disminuir el impacto ambiental negativo y, sobre todo, fortalecer las capacidades de los productores.

Palabras claves: agricultura de conservación, investigación participativa, capacidades, escuelas de campo. 


\title{
Caracterización social, productiva y cultural de la familia campesina en el corregimiento tres del municipio de Villavicencio (Colombia)
}

\author{
Pacheco Pérez Camilo Ernesto MVZ. Esp. \\ Grupo de investigación: GIOVUCC Universidad Cooperativa de Colombia Sede \\ Villavicencio \\ camilo.pacheco@campusucc.edu.co
}

\section{RESUMEN}

Introducción. Confinada a la subregión del piedemonte, históricamente la familia campesina (pequeño productor rural) en los Llanos Orientales de Colombia, ha desempeñado un rol muy importante en el desarrollo integral de esta región, cuya principal vocación agropecuaria ha permitido el establecimiento de cultivos empresariales tanto semestrales como perennes y una ganadería en grandes extensiones de tierras, que permitiría definir las características sociales productivas y culturales propias de esta población. Sin embargo los múltiples cambios en las políticas económicas, sociales y culturales, han variado sustancialmente este territorio, debido principalmente a diferentes factores y dinámicas las cuales son necesarias e importante estudiar; la falta de conocimiento académico y gubernamental acerca de las características reales de las familias con permanencia rural en especial de la subregión del piedemonte Orinocense, generan vacíos sustanciales que redundan en estrategias de acompañamiento sociales, productivas y culturales muy débiles, y en la mayoría de los casos no existen. Objetivos. Desde esta perspectiva, se quiso caracterizar las familias campesinas en un corregimiento del municipio de Villavicencio ( 1 de 8), para analizar como línea de base sus realidades e identificar qué elementos inciden en el comportamiento organizacional y empresarial de las familias del sector rural, que a su vez afectan el desarrollo local en el municipio de Villavicencio; presentado en el trabajo de investigación mencionado y que genera una primera intensión para posterior profundización en el tema.Metodología. La población total fue finita y estuvo delimitada por los corregimientos, del municipio de Villavicencio con actividad agropecuaria asociada a vivienda rural, que referido del total del territorio, presenta esta característica un $69.6 \%$, para un aproximado 
de 2.842 unidades distribuidas por los diferentes sectores con vocación agrícola en los corregimientos del municipio de Villavicencio.Por lo extenso del área rural de cada corregimiento la caracterización de estudio se realizó sobre uno de ellos (corregimiento 3) escogido al azar, considerando que no todos los corregimientos poseen las mismas características, sino que es mejor realizar una caracterización por cada uno. El carecer de fuentes que precisen el número de familias por corregimiento convirtió la población objeto estudio en infinita. El tamaño de la muestra para el diseño de encuesta se basó en una muestra aleatoria simple, calculándose un total de población a encuestar de 350 familias en todo el corregimiento compuesto por 5 veredas para un total por vereda de 70 familias, identificándose un representante por cada una, a quien se le realizaron las preguntas contenidas en el instrumento (encuesta) integrada por preguntas desde la perspectiva social (7 preguntas), productivo (14 preguntas) y cultural (11 preguntas) para un total de 32. Conclusiones. Desde los resultados obtenidos en esta investigación se puedo inferir, en general, las necesidades más sentidas de las familias del corregimiento 3 del municipio de Villavicencio, así mismo y teniendo en cuenta que el tema y que las necesidades desde los componentes social, productivo y cultural interrelacionados, se originan especialmente desde la toma de decisiones político administrativas; enmarcadas por ejemplo desde los planes de desarrollo y planes de ordenamiento territorial para potencializar el desarrollo endógeno local; el autor de este trabajo propone como estrategia sociopolítica, la creación de una OFICINA INTERDISCIPLINARIA DE INVESTIGACIÓN LOCAL, en la Alcaldía Municipal de carácter permanente, creada por proyecto de acuerdo y conformada por un equipo técnico que convoque la participación de funcionarios del Instituto Colombiano de Bienestar Familiar (ICBF), las Universidades, Oficina de planeación municipal, Secretaria de infraestructura, Empresa de Acueducto y Alcantarillado de Villavicencio, la Asociación de Campesinos y el Consejo Territorial de Planeación de Villavicencio Colombia. Lo anterior en cumplimiento de los objetivos propuestos en este proyecto de investigación.Sin embargo se hace importante estudiar los demás 
corregimientos, para después tener la posibilidad de correlacionar variables y profundizar en un tema coyuntural que además ha sido débilmente investigado.

Palabras Clave: socioeconomía productiva, veredas Villavicencio 


\title{
Tendencias y alcances del comercio justo en Villavicencio
}

\author{
Ruiz López Adriana Isabel \\ Administradora de Empresas Agropecuarias - CORHUILA. Esp. Alta Gerencia y \\ Economía Solidaria - UCC, Aspirante MSc Gestión Ambiental Sostenible - \\ UNILLANOS, Universidad Cooperativa de Colombia UCC \\ adrisaruiz@gmail.com
}

\section{RESUMEN}

Introducción. El Comercio Justo promueve relaciones comerciales alternativas basadas en valores como la igualdad, consumo responsable y cuidado del medio ambiente, diferenciándose de prácticas comerciales tradicionales fomentadas en el desequilibrio económico. El aporte de la investigación brinda una herramienta de información y análisis de las tendencias en Villavicencio, permitiendo identificar la aplicabilidad y ventajas del comercio no convencional, surgiendo el interrogante de ¿Cuáles son las tendencias y los avances teóricos divulgados sobre el Comercio Justo en Colombia Caso Villavicencio? Objetivo. La investigación tiene como propósito elaborar una síntesis de conocimiento que permita clarificar las tendencias y alcances teóricos divulgados sobre Comercio Justo en Villavicencio, por medio de la descripción de prácticas no convencionales, antecedentes, diferencias comparativas y el impacto producido en el sector económico de las grandes superficies. Metodología. Enmarcada dentro del tipo de investigación descriptiva, la población objeto de estudio correspondió a las personas que realizan prácticas comerciales en la ciudad de Villavicencio, muestreo de carácter probabilístico a noventa y nueve comerciantes encuestados, dos expertos en el tema y tres directivos de almacenes de cadena de la ciudad de Villavicencio, quienes manifestaron sus experiencias mediante la técnica de entrevista. Resultados. Se encontró que el concepto de Comercio Justo no es reconocido para la comunidad de comerciantes de Villavicencio, confundido con sana competitividad entre los actores comerciales, demuestra que el comercio convencional no tiene un equilibrio e igualdad en la práctica. También, se logró establecer que sus principios se enfocan en el pago de precios justos a los productores directos, con el fin de que las utilidades no se reviertan a 
intermediarios sino para el sustento familiar; concepción dada por la lógica de los enunciados, que por conocimiento de la temática. El consumidor no tiene claro su rol sobre el consumo responsable y su impacto ambiental, aunque toma fuerza el factor de origen de los productos y los procesos de elaboración, aunque no se reconozcan temas de certificación. El conocimiento de un experto como actor activo del Comercio Justo en Colombia, apropia elementos y los ajusta a las necesidades específicas creando una cadena que beneficia a las comunidades participantes; para el experto en Marketing Tradicional, se basa en obtener el máximo beneficio, adecuado dentro de la responsabilidad social de los productos y servicios generados, donde el productor normalmente es el que menor retribución obtiene y el consumidor final acarrea con los costos acumulados en cascada. Los resultados expuestos evidencian que la tendencia del desarrollo del Comercio Justo en el Caso de Villavicencio, avanzará de forma lenta y sin actores definidos que lo promuevan; la práctica comercial no ha logrado estrategias de difusión de la información que conlleve a un mayor nivel de reconocimiento en la población. Conclusiones. El Comercio Justo no sólo permite mayor participación en el comercio de grupos socioeconómicos desfavorecidos de difícil acceso al mercado, sino también la producción con calidad de manera respetuosa con el entorno medio ambiental. Es importante la iniciativa y participación de expertos en el tema en nuestro país, específicamente el enfoque de un comercio justo basado en comunidades participantes en toda la cadena de abastecimiento, brindando una nueva alternativa para la producción y comercialización de los productos. A nivel del Juicio de los expertos se logra evidenciar una nueva tendencia a nivel de Colombia, pensadas a nivel global y propuestas a nivel de agrupaciones aisladas, se evidencia que no hay un bloque conjunto que acelere su promoción y rápido avance.

Palabras Clave: Comercio Justo, tendencias, alcances, productores, consumidores. 


\section{Proyecto de investigación en seguridad alimentaria del centro de investigaciones ambientales - CIAM- del Parque Metropolitano María Lucia

\author{
Rodríguez Arcila Luis Guillermo \\ Profesor Tiempo Completo Dedicación Exclusivalnvestigador CIAM. UNIMETA \\ Profesor investigador CIAM. Médico Veterinario y Zootecnista - Unillanos. \\ Especialista en Ecología y Medio Ambiente - Unimeta Especialista en Acuicultura \\ - Aguas Continentales - Unillanos. \\ luis.rodriguez@unimeta.edu.co
}

\section{RESUMEN}

El Parque Metropolitano María Lucia es un espacio de terreno de 114 hectáreas donado y cobijado bajo la figura jurídica de una Reserva de la Sociedad Civil, en donde se adelanta investigación básica y aplicada con miras a cumplir con procesos de Responsabilidad Social Empresarial por parte de la Corporación Universitaria del Meta UNIMETA. Está situado en el piedemonte llanero a 14 kilómetros de Villavicencio en la Vereda denominada la Llanerita. Desde el año 2010 el señor rector y fundador de nuestra universidad el Doctor don Rafael Mojica García visualizó que en un futuro y con el crecimiento urbano de la ciudad dentro de pocos años debería existir un espacio en donde la gente pudiese tener un sitio de recreación, sano esparcimiento y producción de legumbres y hortalizas para sostener un nivel de vida de acuerdo con las necesidades básicas nutricionales. El proyecto de seguridad alimentaria nace como iniciativa de los estudiantes y el profesor de la cátedra de agroecología de la carrera de Ingeniería Ambiental de proveer de algunos alimentos de consumo diario a los moradores de la región y que debido a los diferentes procesos de cambio social se han dejado de producir en cada una de las fincas del piedemonte llanero. Se estableció un área de 15 por 30 metros cercada en malla para galpón de 1.50 metros de alto con postadura de madera para su aislamiento, se desmatonó y picó el suelo para formar surcos dejando calles de 50 centímetros entre surcos, se procedió a sembrar algunos semillas empacadas compradas en el mercado local, el resultado fue desastroso, la gran mayoría no nació y las que lo hicieron murieron sin llegar a fructificar. Como se trata de ensayos pedagógicos, se plantearon varias hipótesis a saber: las semillas no tenían el poder de germinación y no estaban adaptadas a 
esta región, el suelo no tenía la cantidad de nutrientes necesarios para este tipo de producción, el paquete tecnológico no era el adecuado o sería una combinación de todos los anteriores. Se realizó un análisis de suelos y arrojó unos resultados en donde todos sus componentes estaban entre la categoría de bajo y muy bajo, en cuanto a las semillas se optó por conseguir las que ya están adaptadas al medio y se empezó a realizar recorridos por las fincas adyacentes y recolectar este tipo de material para reproducirlo haciendo énfasis en la forma autóctona en que se cultivaba donde se encontró para así tratar de adecuar un paquete tecnológico de fácil replicación. Para mejorar la cantidad de nutrientes en el suelo se efectuó una actividad que es la elaboración de abonos orgánicos y se empezó a producir compostaje a partir de bovinaza, pollinaza y desechos de la granja, encontrándose después de dos años de ensayo error con un producto de excelentes condiciones nutricionales para las plantas y de un costo muy bajo. Las semillas se recogieron de plantas en producción de las fincas aledañas y se plantaron bajo los mismos principios técnicos del saber popular de las personas en cada uno de los sitios donde fueron obtenidas. Hoy en día se cuenta con plantas aclimatadas, adaptadas y produciendo semillas fértiles en piedemonte llanero entre las que se encuentran las siguientes: Espinaca crespa, Tomate Ilanero, Espinaca morada o Carurú, Pimentón, Coles, Cebolla de huevo, Ajo de hoja, habichuela medio metro, Culantro, Pepino cohombro, Cebolla junca, Maní, Cilantro, Frijol y Maíz. Para solventar las necesidades de proteína de origen animal se inició un proyecto de recuperación genética de la gallina de huevo azul en la cual ya se cuenta con un piedecria seleccionado y los ejemplares $F_{1}$ se encuentran en levante y próximos a iniciar postura. Toda la investigación que es básica y aplicada se lleva a cabo como un proceso de responsabilidad social empresarial de la Unimeta con miras a tener una soberanía alimentaria para los moradores del piedemonte llanero.

Palabras clave: Seguridad alimentaria 


\title{
Experiencia de desarrollo curricular en el campo de las ciencias agrarias
} sistemas agrarios de producción agrícola de clima cálido -SAPACC curso del IX de ingeniería agronómica desarrollado en la unidad rural del Tahúr y la Banqueta

\author{
Álvarez Socha Álvaro ${ }^{1}$ \\ 1 Ingeniero Agrónomo, Docente UNILLANOS, Facultad de Ciencias Agropecuarias \\ y Recursos Naturales, Escuela de Ciencias Agrícolas, Programa de Ingeniería \\ Agronómica, Coordinador Académico IX semestre. \\ alvarsocha@hotmail.com
}

\section{RESUMEN}

Introducción. El programa de Ingeniería Agronómica de la Universidad de los Llanos a partir del análisis de los desarrollos agrícolas que se están dando en la Orinoquia Colombiana crea el curso práctico "Sistemas Agrarios de Producción Agrícola de Clima Cálido" -SAPACC- de régimen especial reglamentado por el Acuerdo Académico $N^{\circ} 005$ de 2010 que se le ofrece a los estudiantes de noveno semestre, a desarrollarse en la unidad rural del Tahúr y la Banqueta, localizada en el Municipio de Villanueva, departamento del Casanare. Los estudiantes realizan allí prácticas académicas rurales, iniciando con ello, un flujo de intercambio de aprendizaje con profesores, académicos y cultivadores de la zona. Los futuros profesionales, adquieren las herramientas técnicas necesarias para el desempeño propio de la carrera. Ésta experiencia inicio en el primer semestre del 2009 con nueve estudiantes, quienes abrieron el camino académico para el desarrollo de una apuesta innovadora en el medio de la educación superior colombiana. Metodología y resultados. EI curso SAPACC busca vincular al estudiante a los procesos integrales de producción agrícola de clima cálido, dotarlos de herramientas necesarias que le permitan el mejoramiento de la producción y la productividad de la unidad rural, que logren una mayor interrelación con la comunidad circundante, se desarrolla en calendario académico de veinte semanas en coherencia con el agrícola de la región; diez y seis en la unidad rural y las cuatro restantes en la sede Barcelona. Se maneja un manual de convivencia, un gobierno estudiantil y acompañamiento de un Ingeniero residente. El curso 
reproduce las condiciones, en tiempo real, de desarrollo de los proyectos productivos, lo cual implica el manejo agronómico desde la planeación y conducción del cultivo, situación que obliga a la participación del estudiante en la toma de decisiones y su implementación. Los componentes académicos que se desarrollan son: pecuarios, cultivos semestrales, cultivos perennes, seguridad alimentaria todos enlazados a la extensión rural y al cierre de semestre se realiza un seminario con temas de interés de la comunidad. Como resultado tenemos: doscientos treinta y nueve estudiantes que han aprobado el curso, nueve seminarios con sus respectivas cartillas, mejoras en el nivel de vida de los campesinos; se ha sembrado arroz, soya y maíz, en especies perennes Plátano, Yuca, Cacao, en seguridad alimentaria, cultivos de tomate, habichuela, pepino, maíz, pimentón y otros con resultados satisfactorios. La extensión rural se da a partir de una agenda de visitas en las cuales se identifica la problemática, se toman elementos de discusión con los cuales se concluye y se recomienda en un intercambio de saberes.

Palabras claves: SAPACC, semestrales, perennes, seguridad alimentaria, seminario, extensión rural. 